\title{
Documentation and First Data-Analysis on Acceptance Tests of W7-X Coils
}

\author{
K.Hertel $^{1}$, J.Baldzuhn ${ }^{1}$, H.Viebke ${ }^{1}$, G.Croari ${ }^{1}$, L.Genini ${ }^{2}$, R.Bertrand ${ }^{2}$, L.Vieillard ${ }^{2}$, H.Ehmler ${ }^{3}$
}

(1) Max-Planck-Institut für Plasmaphysik (IPP), Greifswald Branch, Euratom Association, Wendelsteinstraße 1, D-17491 Greifswald, Germany

(2) Commissariat à l'Energie Atomique Saclay (CEA), F-91400 Gif sur Yvette, France

(3) Hahn-Meitner Institut Berlin (HMI), Glienicker Str. 100, D-14109 Berlin, Germany

Author's contacts: $\quad$ IPP, Greifswald (Gemany) Tel.: +49 / 38.34 / 8823.68

CEA, Saclay (France) Tel.: +33/ 169.08.57.06

E-mail address: kerstan.hertel@ipp.mpg.de

CEA contacts: $\quad$ Laurent Genini, CEA, Saclay (France) Tel.: +33 / 169.08.22.27

E-mail address: genini@dapnia.cea.fr

Laurence Vieillard CEA, Saclay (France) Tel.: +33 / 169.08.95.75

E-mail address: vieillard@dapnia.cea.fr

\begin{abstract}
The WENDELSTEIN 7-X (W7-X) superconducting stellarator experiment i.a. consists of 50 non planar coils (npc) and 20 planar coils (plc). Before assembly the function of every coil is tested and examined at the French partner-institute CEA, Saclay.

The testing follows a certain and constant routine laid out in a master-document (overview); the main characteristical results are then recorded in appropriate reports. This presentation will point out the importance of good documentation, give an example of how it might be organized and show its respective needs of time and manpower.

After testing some of the main data is collected in various tables and analysis-software for a first assessment and statistical analysis. The results will be presented as an example of a comparative first-step-database.
\end{abstract}

\section{Contents}

1) Introduction

2) Structure of Test Procedure

3) Structure of Documentation

Document-Types

i. Overview

ii. Test Reports

iii. NCRs

iv. Photos

4) Conclusion and Upshot

5) Bibliography

\section{1) Introduction}

The WENDELSTEIN 7-X-Project will be realized by the Greifswald branch of the Max-Planck-Institute for Plasma Physics Garching. This project is a superconducting stellarator-type fusion experiment and amongst others consists of 70 superconducting coils of different types. These types are distributed as follows:

- 20 planar coils, i.a. used for plasma-positioning, respectively 10 planar coils of type A (1) and type $\mathrm{B}(2)$.

- 50 non planar coils creating the magnetic vessel; respectively 10 non planar coils of type 1 , type 2 , type 3 , type 4 and of type 5 .

Before the coils are installed into the machine, it has to be clarified if they work in accordance to specified requirements. This is necessary because of the complex shape of the non planar coils and the technical 
challenges in general, for example cryogenics vs. leakages or superconductivity vs. high-voltage. These testspecifications are mainly the current which a coil has to carry, the voltage the insulation has to sustain and the maximum accepted amount of helium which leaks out of a coil. The thresholds of the above mentioned specifications are:

- Current at superconductivity (at B and T): planar coils $I=16 k A$, non planar coils $I=17,6 k A$;

○ DC insulation resistance: $R>500 M \Omega$;

○ Leak rate in cryogenic conditions: $q_{L}<1 \cdot 10^{-7} \frac{\mathrm{mbar} \cdot \mathrm{s}}{\mathrm{s}}$.

The current has to be reached in self-field (B) and temperature T, $0.1 \mathrm{~K}$ smaller than quench-temperature. For that purpose every coil is tested in a cryogenic test facility, provided and driven by the Commissariat à l'Énergie Atomique (CEA) in Saclay, France. Finally, the results of these tests are recorded in respective documents.

\section{2) Structure of the Test Procedure}

The test-program of the coils at CEA is based on a parent test instructions called Scope and Requirements for the Acceptance Tests on the W7-X-Coils [1]. These instructions were created by taking into account information and values from the coil-specifications Technical Specifications of npl-Coils [2] and Technical Specifications of plCoils [3]. On the basis of these instructions a certain test-structure was created, which considers the instructions' requirements as well as the technical abilities of the test facility at CEA and the work cycle for the necessary assemblies and installations. This general structure is shown in the following figure:

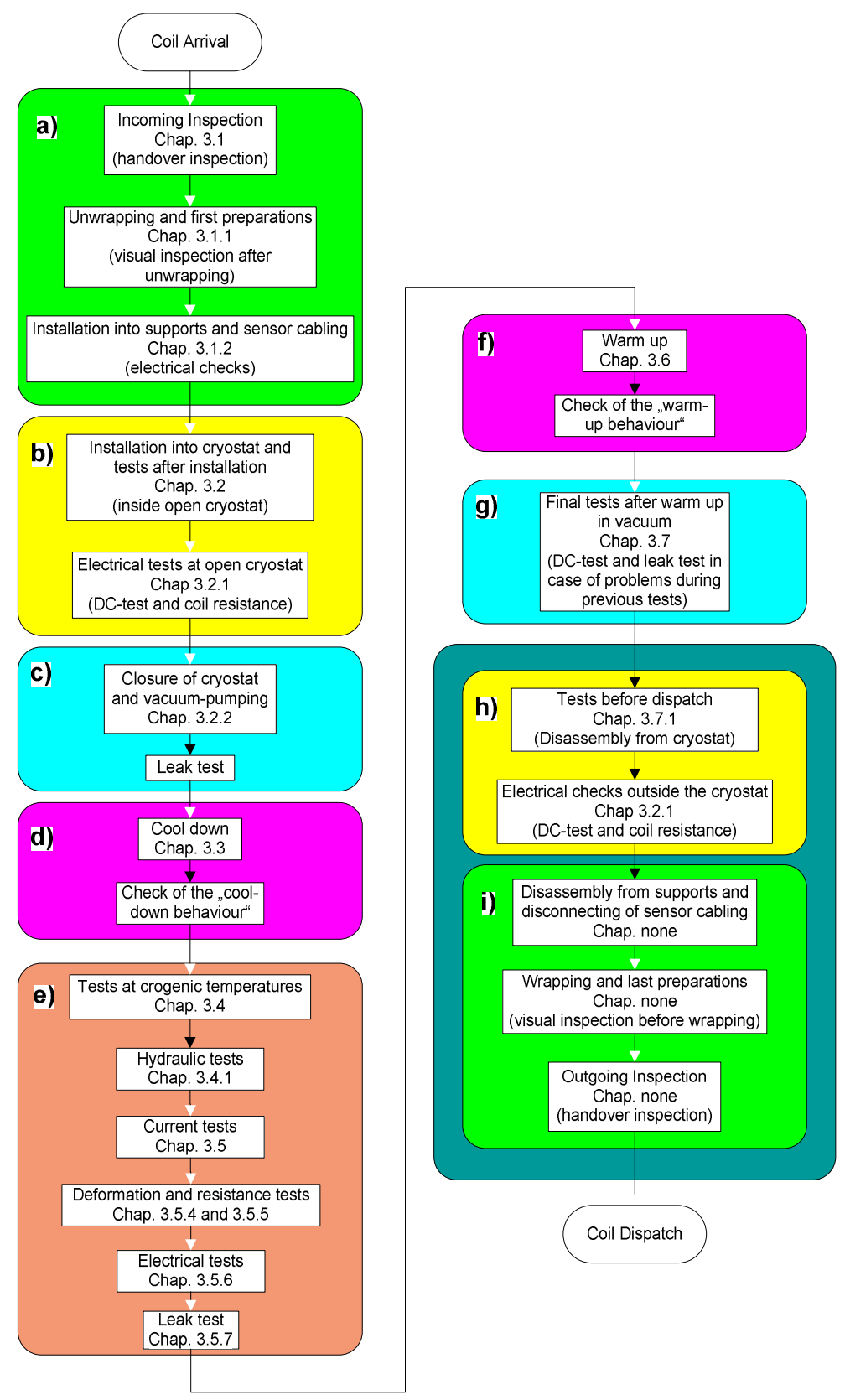


Consequently, the whole test procedure is subdivided into separate chapters due to the condition in which a coil is located. These conditions are i.e. the location, the present status of assembly or the environment (inside a cryostat at RT, but in vacuum). All chapters which are mirror-images of previous ones are coloured equally, except the one describing the cryogenic test. Therefore, test results in chapters with the same colours should be the same. The last chapter is in fact two separate chapters, because the work-cycle-conditions implicate two different steps in the test procedure. This could be interpreted as an uncertainty of the structure.

\section{3) Structure of Documentation}

The documentation has to follow the structure described above and is divided into chapters as follows:

- Incoming inspection (on transport frame, assembly into test-support)

- Tests after installation (open cryostat, coil mounted in support and everything inside the cryostat)

- Tests in closed cryostat (ambient temperature, vacuum)

- Cool down

- Tests at cryogenic temperature

- Warm up

- Final tests after warm up (ambient temperature, vacuum)

- Tests before dispatch (disassembly from test-support, putting onto transport frame)

\section{Document-Types}

The documentation is usually carried out according to four different records/files:

- Overview, (the main document),

- Test Reports, (including selected data),

- NCRs, (describing failures),

○ Photos.

Besides that, the documentation may also include documents describing irregularities or exceptional tests which are then labelled as miscellaneous documents in miscellaneous folders. It is advisable, to store all documents in a compelling folder system which must not be changed during the whole test project (following figures).

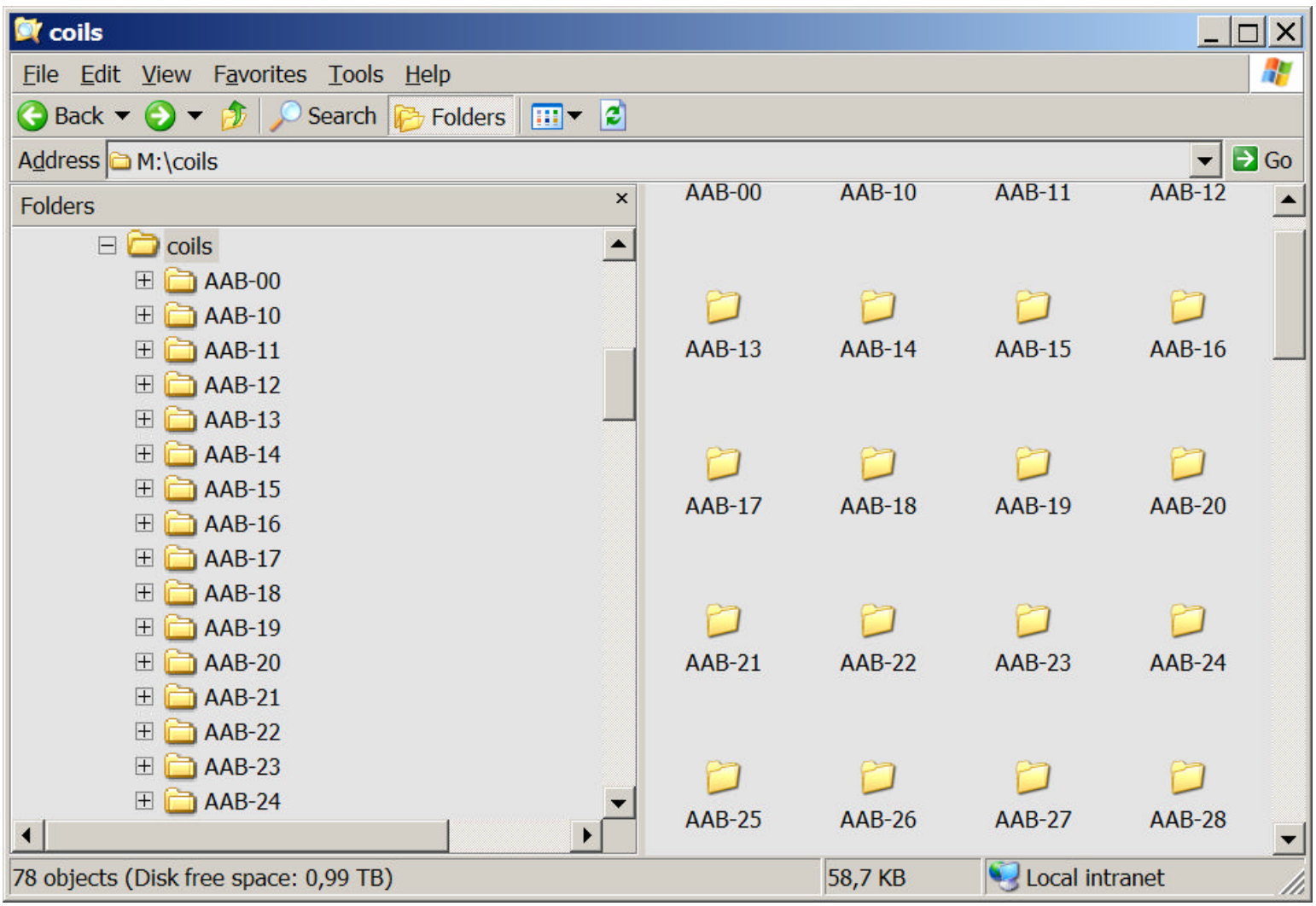




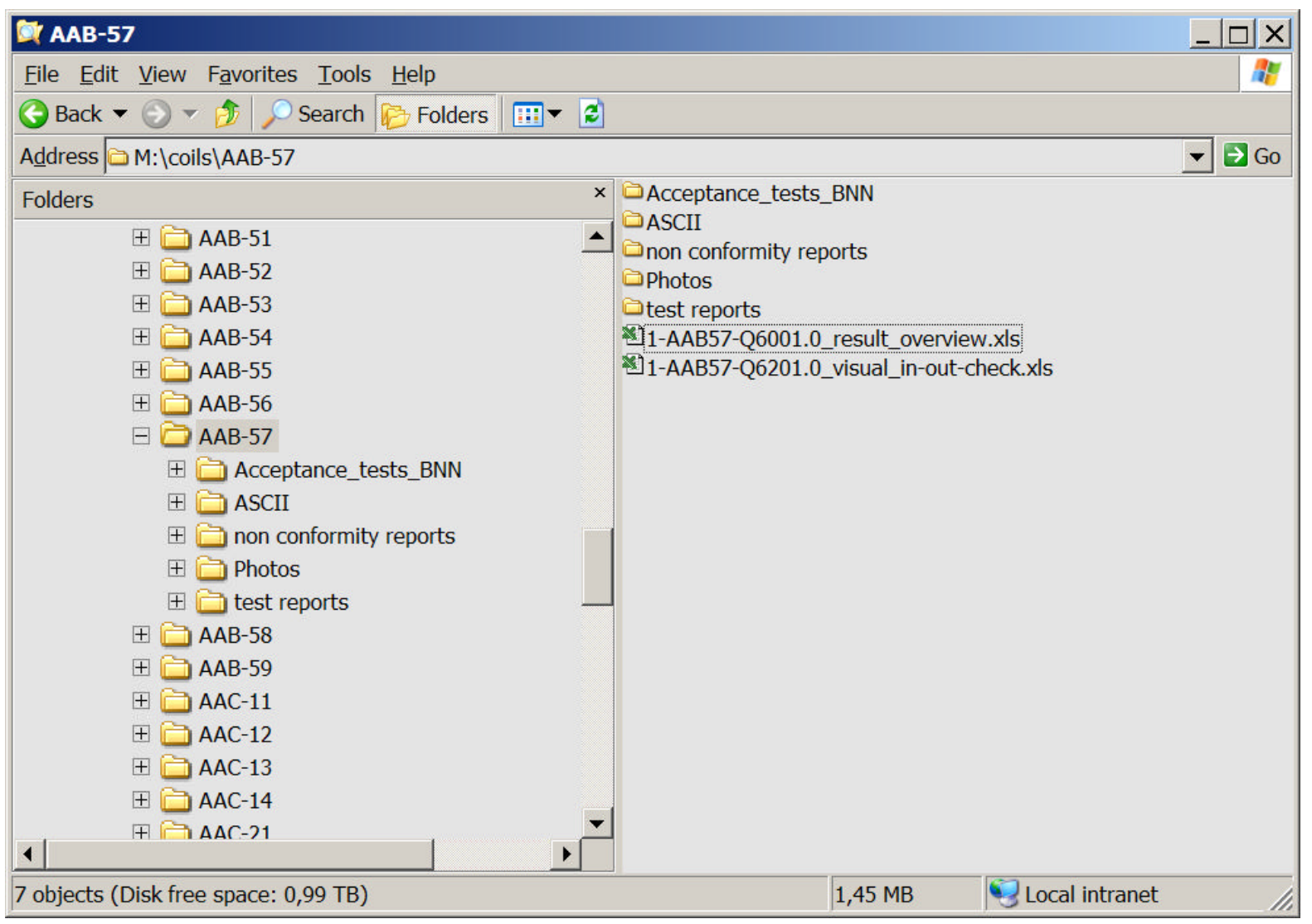

It is necessary to abide by this folder-system in order to maintain all document-automatics and to avoid too much manual work. This is especially important for the document explained below.

\section{i) Overview}

This document is the central part of a coil-test-documentation and contains three different worksheets. The first is the actual Overview which is organised according to the structure in the flowchart explained above. It includes the main information like the coil type, which coils were tested together, in which cryostat it was tested, the test duration, most of the main values and, besides others, all test reports and NCRs as hyperlinks. So, they can be opened directly from the overview. The second worksheet is the so-called Handover Certificate which has to be filled in when a coil arrives or leaves the test facility. It is a record of the arrival/dispatch conditions and describes how the coil is loaded onto the truck, if the plastic foil in which the coil is wrapped is damaged, or the conditions of the shock sensors.

Because the coil is wrapped in foil during this check it is difficult or even impossible to verify the coil's condition itself. For that reason, a third worksheet was created, which is called Inspection Unwrapped Coil and has to be filled out, when a coil was unwrapped to prepare it for testing or when a coil will be wrapped for the dispatch. This table serves as a checklist for the coil-condition like residuals on the casing, damaged sensors or oxidized strand surfaces. Each coil is kept wrapped until shortly before the test preparations to avoid any soiling during the storage at Saclay. Therefore some time may pass between the completion of the Handover Certificate and that of the Inspection Unwrapped Coil worksheet.

The Overview-file contains a certain functionality to avoid repetitive manual work and to simplify the issuing of a new Overview-file for a different coil. Moreover, it includes a safety precaution that prevents that a test might be skipped and it points out if a value exceeds its limits. To keep it clearly arranged necessary tables can be created just when they are needed by pushing a button. The following screenshots may give a glimpse onto the shape of the Overview's worksheets. 


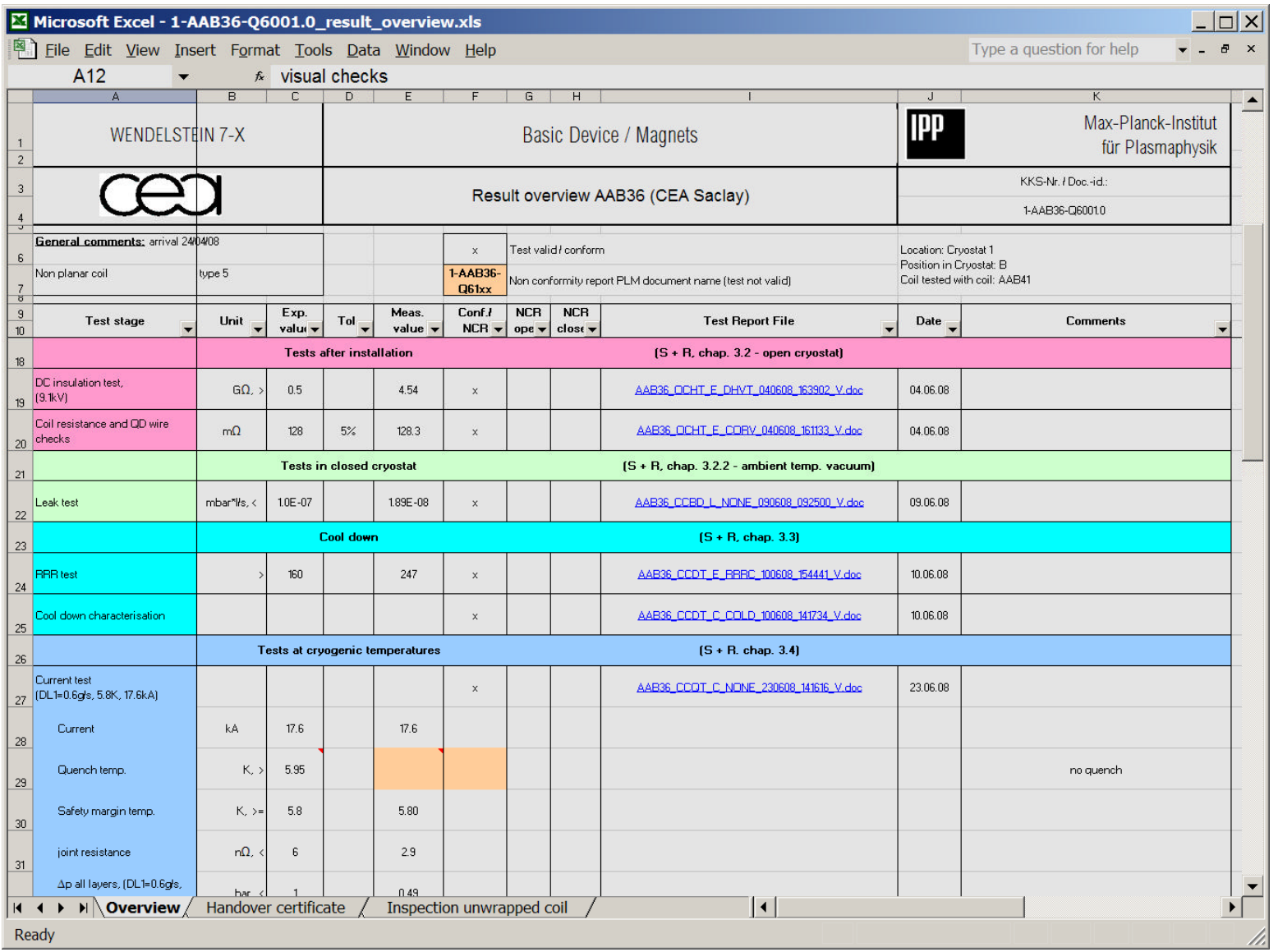

Screenshot of Overview-table

区icrosoft Excel - 1-AAB36-Q6001.0_result_overview.xls

囷 File Edit View Insert Format Iools Data Window Help

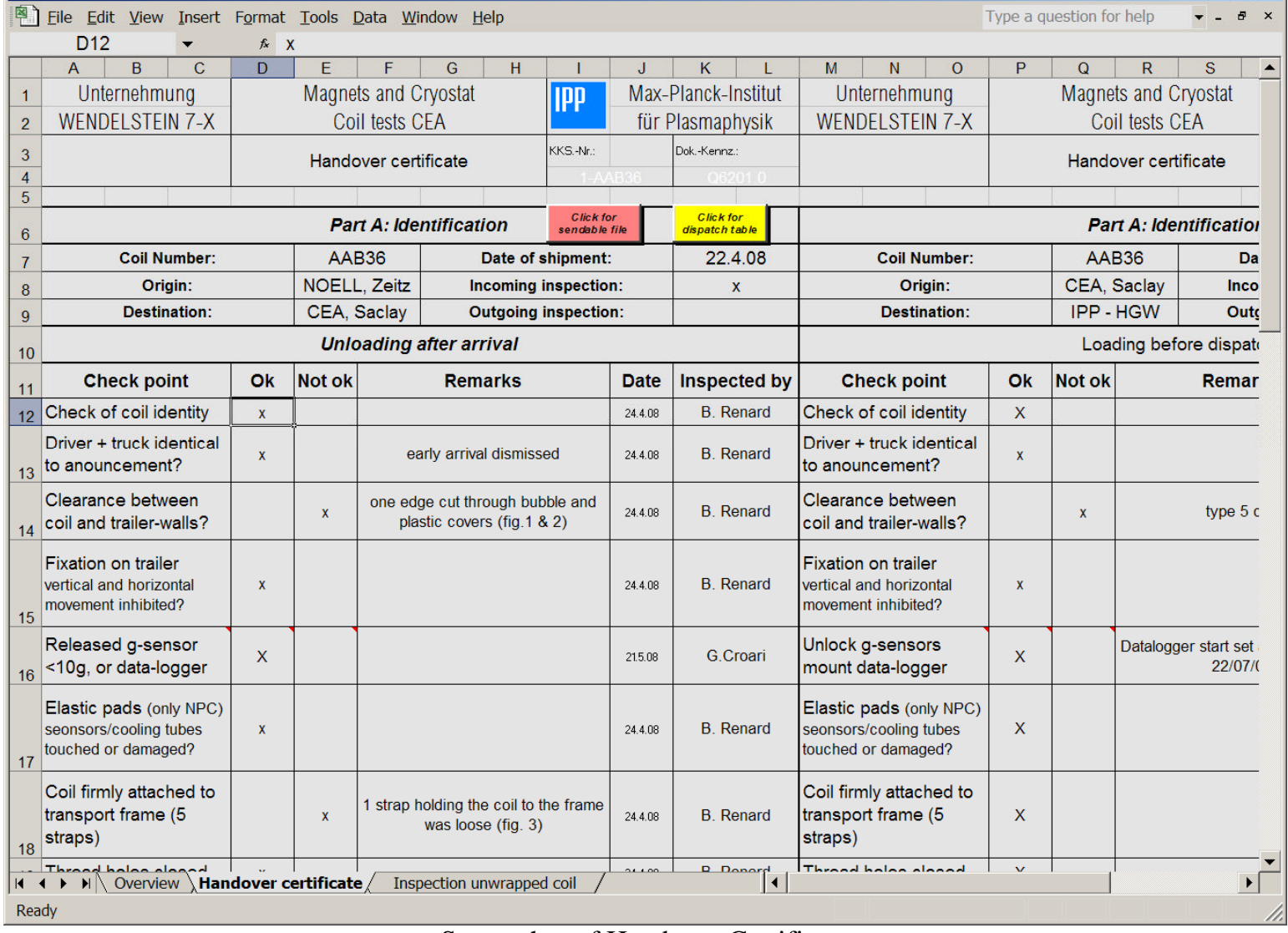

Screenshot of Handover Certificate 


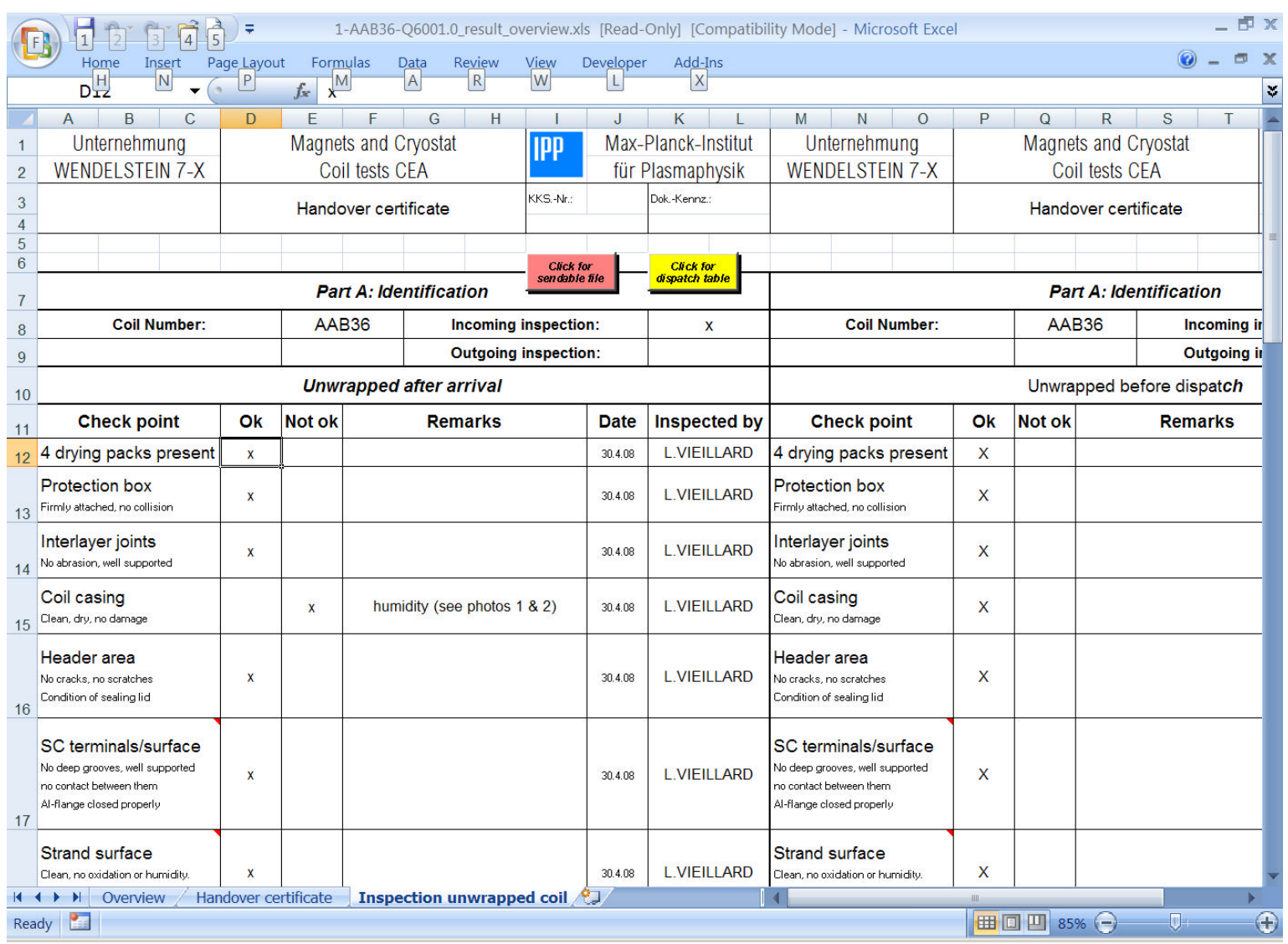

Screenshot of Inspection after Arrival

\section{ii) Test Reports}

These documents collect the conditions in which a test had taken place. They include respective data and curves and the test result and are simply a description as complete as possible of the whole test. Nevertheless, they might not include all data, signals and curves. As a consequence, these reports have to be interpreted as the first analysis of the sample-tests. Their extent depends on the agreed document-tasks, the manpower which is available for documentation and the time until a test report has to be finished. Especially when problems have occurred, it could take some more time to finish a report.

If the signals and curves cannot be presented completely, only the most important data has to be considered and shown in the report. For example, in the W7X-test project the data and signals (of sensors) which are meaningful or/and critical were selected, data with a poor reliability was skipped. Selected data and signals are i.e.:

- Insulation and wire-resistances of QD-cables, strain gauges, extensometers, temperature-sensors, and their harnesses before installation into and after removal out of the cryostat,

- $\mathrm{AC}$ and $\mathrm{DC}$ tests in open cryostat before and in closed cryostat after current tests,

○ He-background and He-leaks in cryostat at vacuum,

○ RRR- and temperature measurement during cool down and warm up,

- He-Pressure, He-mass flow, Temperatures, voltages and current during current test,

Especially during the current tests some data was neglected because of their poor reliability. These were i.e. the extensometers which often failed completely and showed meaningless results, as well as the strain gauges showing data which was not completely understood at first. This data was investigated further after the tests were finished.

The different types of test reports are divided in the same way as the above mentioned chapters. This is considered and labelled in the filenames of the test reports (as well as the date and the time). For example a current test has been coded as follows:

AAB33_CCQT_C_NONE_230608_141616_P.doc

- AAB33 - Non Planar Coil 33, type 3 (planar coils are coded with C instead of B),

- CCQT - Closed Cryostat Cryogenic Temperature (superconductivity),

- C - Current test,

- NONE - Placeholder for other codes like DHVT which means Direct High Voltage Test,

- 230608 - Date (day month year)

- 141616 - Time (hour minute second)

○ P $\quad-$ Problem (test not successfully passed in all points; instead V would indicate Valid) 


\section{iii) NCRs}

In case of a malfunction or any other unusual event these documents will be created. In which, it is insignificant if the event or malfunction was caused by the facility, an external measurement-device or by the coil itself. The reason is that due to the schedule normally the tests cannot be repeated or at best only during a certain time. These tests might be accepted with the available result or sometimes have to be skipped. An NCR is always related to the respective test report. Therefore, a test report may be mentioned several times in the Overview, depending on the amount of related NCRs. The description of the malfunction is mostly the same as in the test report and added only with solution-ideas and agreements between the two institutes, whereas the test reports include only the technical descriptions.

An NCR can be classified as "Minor" or "Major". A classification as "Minor" means that the malfunction will not affect the machine itself. Mostly these are NCRs about malfunctions in external measurement-devices, like LCR-meters, which did not work properly or a cord grip which was missing. A "Major" NCR describes a malfunction which might affect the machine, like He-leaks or broken temperature sensors. Usually, these NCRs need a further investigation.

\section{iv) Photos}

Photos are much more important than initially expected, especially to explain technical issues to people or colleagues who are not familiar with the test project or with the daily work routines. Additionally, every malfunction, defect or irregularities shall be documented with a photo in general. This can then serve as a reminder and evidence when these topics are discussed later. In this context, it has to be mentioned that it is not the amount of photos which is important, but the perspective and the exposure-quality. A photo which is unfocussed or has an incorrect exposure is useless. Because of that the author of the documents, who normally does all the photos too, needs a convenient equipment and an understanding of how to take technical photos. Some of the main points which have to be considered are:

- For good orientation, the first photo has to be a panoramic overview (before taking a zoomed-in detail-photo). Alternatively, the location of it might be noted (with a marker) directly onto the detail of the sample.

- The top of a zoomed-in detail must be the top in the photo. Otherwise, orientation will be difficult. If the details are horizontally orientated, the top of the photos should be in northern direction or twelve o'clock of the sample.

- A direct flashlight is useless, if the sample has metallic surfaces which may reflect strongly. In case of less light an additional photo-spotlight or external indirect flashlight might be necessary.

- If the exposure-time is long, a tripod and a remote shutter is needed.

- Sometimes, a good depth of field is very important in technical photos. To achieve this, the aperture has to be small. As a consequence, a good exposure might be difficult, because a lot of light is necessary.

Often, a picture speaks louder than words.

\section{4) Conclusion and Upshot}

A serial documentation of a component-test needs quite a lot of skilled manpower and should not be underestimated. To achieve successful research, maybe years later when the staff might have changed, one depends on the quality and comprehensibility of the documentation. It can be pointed out that the knowledge of a project will disappear with the staff that leaves, if the documentation cannot serve as a kind of knowledgereservoir.

The structure of the present documentation was developed during the coil tests and led partly to different coiltest-documentation. This will complicate a comparison between the same tests of different coils and should be avoided in similar projects. The structure should be established and proved before the real project starts. The present documentation of the coil tests in Saclay contains a certain automatic system to help issuing an Overview when a new coil arrives. But there is still a lot of repetitive manual work to do. To further simplify this, models for every type of test report should be created. Furthermore, it has to be assured that the work will not be constrained by manuals and instructions which are difficult to understand. Especially within an international cooperation, where people usually have to communicate in a language which is not their mother-tongue, the misunderstandings are prone to occur.

\section{5) Bibliography}

[1] Scope and Requirements for the Acceptance Tests on the W7-X-Coils

[2] Technische Spezifikation für das Nichplanare Spulensystem des WENDELSTEIN 7 - X Experimentes

[3] Technische Spezifikation für das Planare Spulensystem des WENDELSTEIN 7 - X Experimentes

[4] Chapter-distribution by CEA and IPP.

[5] Development of the Overview by CEA and IPP. The Overview-macros are developed by the author.

[6] Development of the Test Reports by CEA.

[7] Development of the NCRs by CEA and IPP. 